\title{
Non-adherence to chelation therapy and associated psychosocial factors among transfusion-dependent thalassaemia patients in Kandy, Sri Lanka
}

\author{
*Anuradha Baminiwatta ${ }^{1}$, Ruwan Gunathilake ${ }^{2}$, Shiyama Arambepola ${ }^{3}$, Mahinda Arambepola ${ }^{2}$
}

Sri Lanka Journal of Child Health, 2021; 50(1): 116-123

\begin{abstract}
Background: Poor adherence to iron chelation may underlie the detrimental levels of iron overload previously reported among transfusion-dependent thalassaemia (TDT) patients in Sri Lanka. Given the many challenges faced by these patients and families, psychosocial factors may have a significant impact on medication adherence.
\end{abstract}

Objective: To assess chelation adherence among TDT patients and explore associated psychosocial factors.

Method: TDT patients, aged over 4 years, admitted for blood transfusion to a tertiary care hospital, as well as their mothers, were recruited. Medication adherence was assessed using a questionnaire adapted from Brief Adherence Rating Scale. Serum ferritin (SF) values were recorded as objective measures of non-adherence. Mental health status of patients and their mothers was assessed using Strengths and Difficulties Questionnaire (SDQ) and General Health Questionnaire-30 (GHQ) respectively. Associated psychosocial factors were analysed using multiple linear regression.

Results: Fifty two patients aged 4 to 28 years participated. Thirty four $(65 \%)$ were female. The average adherence rate was $90 \%$. One fifth of patients had an adherence percentage less than $80 \%$. The mean ferritin level was $3260 \mathrm{ng} / \mathrm{ml}$, whereas $46.2 \%$ of patients had SF levels exceeding $2500 \mathrm{ng} / \mathrm{ml}$. SF levels correlated positively with non-adherence $\quad(\mathrm{r}=0.34, \quad 95 \% \mathrm{CI}: \quad 0.071-0.606$, $\mathrm{p}=0.014$ ). Based on multivariate analysis,

${ }^{1}$ Department of Psychiatry, Faculty of Medicine, University of Kelaniya, Ragama, Sri Lanka, ${ }^{2}$ Paediatric Unit, National Hospital, Kandy, Sri Lanka, ${ }^{3}$ Psychiatric Unit, National Hospital, Kandy, Sri Lanka

*Correspondence: baminiwatta.akab@gmail.com

D https://orcid.org/0000-0002-5495-2029 (Keceived on 04 April 2020: Accepted after revision on 22 May 2020)

The authors declare that there are no conflicts of interest

Personal funding was used for the project.

Open Access Article published under the Creative

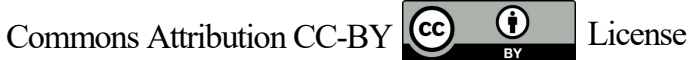

increasing age and lower SDQ score of patient, lower educational level and greater GHQ-30 score of mother were independent predictors of higher SF levels, but not of adherence rates.

Conclusions: In the paediatric unit of National Hospital, Kandy, $46 \%$ of patients had SF levels $>2500 \mathrm{ng} / \mathrm{ml}$. There was a significant correlation between SF levels and medication non-adherence $(\mathrm{r}=0.34, \quad \mathrm{p}=0.014)$. On multivariate analysis, increasing age and lower SDQ score of the patient, lower educational level and greater GHQ-30 score of the mother were independent predictors of higher SF levels, but not of adherence rates.

DOI: http://dx.doi.org/10.4038/sljch.v50i1.9412

(Keywords: transfusion dependent thalassaemia, medication adherence, iron chelation, serum ferritin, psychosocial factors, Sri Lanka)

\section{Introduction}

Thalassaemia is an inherited blood disorder resulting from defective synthesis of haemoglobin ${ }^{1}$. Based on transfusion requirements, thalassaemia is categorized into transfusion-dependent thalassaemia (TDT) and non-transfusion-dependent thalassaemia ${ }^{1}$. Although prevention programmes involving premarital screening have led to a minor attenuation in the incidence, thalassaemia still remains a major health burden especially in low and middle income countries ${ }^{2}$. An island-wide survey in Sri Lanka (SL) has revealed that about 1800 patients with thalassaemia are on regular transfusions and around 50 new cases are born each year ${ }^{3}$. It has been estimated that provisions for thalassemia management may amount to nearly $5 \%$ of the health expenditure in $\mathrm{SL}^{4}$.

Iron overload is the clinically most important complication of thalassaemia, which leads to cardiac failure, diabetes mellitus, hypothyroidism, hypoparathyroidism and hypopituitarism ${ }^{1}$. Therefore, iron overload needs to be minimized using careful monitoring and treatment. With the advent of effective iron chelation medications, iron overload complications have declined, improving life expectancy over the last few decades, especially in high-income countries ${ }^{5}$. However, life expectancy data for Sri Lankan patients are not available. 
Iron chelation was conventionally achieved using parenteral desferrioxamine. Patient compliance for was understandably poor and dictated survival outcomes for several decades, until the introduction of effective oral chelating agents - deferiprone in 1987 and deferasirox in $2006^{6,7}$. Monitoring of body iron status is performed routinely by measurement of serum ferritin at regular intervals. Liver iron concentration is a more accurate indicator of iron status utilized in high-resource settings ${ }^{1}$. Serum ferritin is a useful alternative as it is easy and inexpensive to measure repeatedly, and correlates reasonably well with the body iron burden ${ }^{8}$. Maintaining serum ferritin levels below $2500 \mathrm{ng} / \mathrm{ml}$ has been associated with lower mortality, particularly in relation to cardiac deaths ${ }^{9}$.

Adherence to iron chelation therapy is imperative in TDT, as body iron status determines prognosis 9 . Although the introduction of oral chelation agents revolutionized TDT management, the issue of nonadherence has not completely left the picture. Poor adherence rates have been observed for deferasirox as well, e.g. $42 \%$ of patients displayed poor adherence to deferasirox in a recent Indian study ${ }^{10}$.

Psychosocial factors such as the mental health of patients and their parents, education and financial status may also play a significant role in determining adherence ${ }^{11}$. The stress imposed by regular hospitalization, blood transfusions, as well as concerns related to body image, health and life expectancy would predispose TDT patients to mental health issues. A recently published Sri Lankan study reported that lack of family support, longer duration of hospital stay and female gender were associated with both depression and quality of life among thalassaemia patients ${ }^{12}$. Detrimentally high serum ferritin levels reported previously in SL place these thalassaemia patients at risk of developing iron overload complications at an early age ${ }^{3}$. As psychosocial attributes of these patients are potentially modifiable, insight regarding such factors underlying medication adherence would enable tailoring of strategies to enhance outcomes.

\section{Objectives}

The objectives of this study were to assess 1) the non-adherence to iron chelation and 2) psychosocial factors associated with non-adherence among TDT patients.

\section{Method}

Study design, setting and participants: This was an observational study, conducted at the Paediatric Unit of National Hospital, Kandy. All transfusiondependent thalassaemia patients admitted for their routine blood transfusion, over a period of 3 months, and their accompanying mothers were included as participants. Children $<4$ years and any patients who were acutely ill were excluded.

Measures: A 4-part questionnaire, including background information (Part 1), assessment of medication adherence (Part 2), assessment of the mental health status of the patient (Part 3) and assessment of the mental health status of the mother (Part 4), was used.

\section{Strengths and Difficulties Questionnaire (SDQ):}

Sinhala- and Tamil-translated and validated versions of the SDQ, a 25-item screening questionnaire widely used in mental health research on children over 3 years, was selected as the measure of mental health problems among the TDT patients during the preceding 6 months ${ }^{13}$. For children younger than 11 years, the parent-report version was used, whereas for literate children aged over 11 years, the self-administered version was provided. This questionnaire assesses five areas viz. emotional problems, conduct problems, hyperactivity, peer problems and pro-social attributes using five items for each area. Response to each item is given a score out of 2 . The sum of scores on the first four areas generates a "total problems score" out of a maximum of 40. Although the self-report version of SDQ was originally developed for children aged 11 to 16 years, it has been tested on young adults, demonstrating equivalent psychometric properties ${ }^{14}$.

Medication adherence assessment: To assess adherence to iron chelation therapy, both subjective and objective measures were used. A subjective measure of adherence was obtained by interviewing the patient and the mother separately regarding the amount of chelation medication (both oral and parenteral) taken by the patient during the previous 6 months. For children less than 11 years old, only the mother's report was obtained. The participant's account of the number of days where medication was missed or taken in reduced amounts were critically analysed and the clinician determined the percentage of adherence based on this. This assessment method was adapted from the Brief Adherence Rating Scale ${ }^{15}$. A consultant psychiatrist was involved in designing the interview, to ensure that it was conducted in a nonthreatening and facilitative manner. The first fifteen patients in the study were interviewed by both a consultant paediatrician and a medical officer independently, and their ratings were compared. As the ratings were not markedly different, the medical officer proceeded with the rest of the data collection. The average of the percentages reported by the mother and the patient was termed the 'adherence percentage'. Non-adherence percentage is equal to $100 \%$ minus 'adherence percentage'. As an objective biochemical indicator of the adequacy 
of chelation and an indirect measure of medication adherence, recent serum ferritin levels were recorded. Serum ferritin is routinely monitored once every three months for most patients in this centre. Last measured ferritin level and the level measured 6 months before that were abstracted from patient's monitoring records. The average of the above two ferritin levels was used in analyses. Serum ferritin in our centre is measured using Enhanced Chemiluminescence Immunoassay.

General Health Questionnaire (GHQ)-30: The translated and validated versions of the GHQ-30, a 30 item self-rated screening tool, was used to assess the mental health status of the mothers of TDT patients ${ }^{16}$. GHQ-30 assesses potential nonpsychotic psychiatric disorders and focusses on two main areas i.e. inability to carry out normal functions, and the appearance of new and distressing phenomena. It does not endorse any subscales, but an overall score can be calculated using the sum of scores for the 30 items. Different scoring criteria have been used with the GHQ-30, but for the purpose of this study, Likert scoring method, which was used during the validation of the Sinhala translation ${ }^{16}$, was adopted. Higher GHQ scores indicate a greater degree of mental health problems. Clinical cut-offs for GHQ-30 have not been validated in the local setting so far.
Procedure: The questionnaires were administered to patients (and their mothers) admitted for routine blood transfusions.

Ethical issues: Ethical approval was obtained from the Research and Ethical Review Committee of General Hospital (Teaching) Kandy (ERC No. THK/ERC/08/2019). Informed written consent was obtained from the mothers of all the subjects. For those aged over 16 years, consent of the patients was also taken in writing. No personally identifiable information was recorded.

Statistical analysis: All data were entered into the Statistical Package for the Social Sciences-20 $0^{\text {th }}$ version. Descriptive statistics were reported for both predictor and outcome variables. Multiple linear regression analyses were performed for two outcome variables - non-adherence percentage and serum ferritin level- separately. Statistical significance was set at a p-value of 0.05 .

\section{Results}

Socio-demographic profile

Fifty two TDT patients participated in the study, of whom, $34(65 \%)$ were female. The patients ranged in age from 4 to 28 years, with a mean of 15.5 years $(\mathrm{SD}=7.5)$. The age and gender distribution of the sample is further demonstrated in Table 1.

Table 1: Age and sex distribution of transfusion-dependent thalassaemia patients $(n=52)$

\begin{tabular}{|l|c|c|c|c|c|}
\hline \multirow{2}{*}{ Gender } & \multicolumn{5}{|c|}{ Age (years) } \\
\cline { 2 - 6 } & $\mathbf{1 0}$ or less & $\mathbf{1 1 - 1 6}$ & $\mathbf{1 7 - 2 1}$ & Over 21 & Total \\
\hline Female n (\%) & $09(26.5)$ & $09(26.5)$ & $06(17.6)$ & $10(29.4)$ & $34(100.0)$ \\
\hline Male n (\%) & $07(38.9)$ & $02(11.1)$ & $05(27.8)$ & $04(22.2)$ & $18(100.0)$ \\
\hline Total n (\%) & $16(30.8)$ & $11(21.2)$ & $11(21.2)$ & $14(26.9)$ & $52(100.0)$ \\
\hline
\end{tabular}

With regard to parental level of education, the average number of years of education was 9.5 years $(\mathrm{SD}=3.9)$ for mothers and 10 years $(\mathrm{SD}=2.9)$ for fathers; this difference was not statistically significant $(\mathrm{t}=-1.32, \quad \mathrm{p}=0.193)$. The average monthly income of both parents was 31,000 Sri Lankan rupees $(\mathrm{SD}=18,870)$ whereas the average monthly expenditure attributed to the disease was 4760 rupees $(S D=5209)$. Monthly expenses on treatment, expressed as a percentage of the total income, ranged from $2.5 \%$ to $75 \%$. The distance from the hospital to the patient's residence ranged from $1 \mathrm{~km}$ to $160 \mathrm{~km}$, with a mean of $50 \mathrm{~km}$. Patients had spent on average 14.4 days $(\mathrm{SD}=5)$ in hospital during the last 6 months. Eighteen (35\%) patients had at least one sibling affected by thalassaemia major.

\section{Adherence rates and ferritin levels}

Percentage of adherence to iron chelation over the preceding 6 months ranged from $58 \%$ to $100 \%$, with an average of $90 \%(\mathrm{SD}=10.3 \%)$. Table 2 demonstrates further the different levels of adherence among the patients.

Table 2: Distribution of adherence levels among TDT patients $(n=52)$

\begin{tabular}{|l|c|}
\hline \multicolumn{1}{|c|}{ Adherence level } & n (\%) \\
\hline $100 \%$ & $13(25.0)$ \\
\hline $95-99.9 \%$ & $11(21.2)$ \\
\hline $90-94.9 \%$ & $08(15.4)$ \\
\hline $80-89.9 \%$ & $10(19.2)$ \\
\hline$<80 \%$ & $10(19.2)$ \\
\hline
\end{tabular}

The mean adherence rate for patients who were on subcutaneous desferrioxamine treatment was $82.5 \%$; the corresponding value for those only on oral agents was $91.4 \%$. This difference was statistically significant $(\mathrm{t}=-2.1, \mathrm{p}=0.035)$. Common reasons cited for missing medications were poor tolerability of the drug (34\%) and travelling (27\%). The mean ferritin value of patients was $3260 \mathrm{ng} / \mathrm{dl}$ $(\mathrm{SD}=2112$, Range $=612 \mathrm{ng} / \mathrm{dl}$ to $8810 \mathrm{ng} / \mathrm{dl})$. Table 3 demonstrates further the different levels of serum ferritin values in the sample. 
Based on ferritin values 6 months apart, there was a mean drop of $77 \mathrm{ng} / \mathrm{dl}(\mathrm{SD}=1848)$ over this period, which was statistically non-significant $(\mathrm{p}=0.424)$. $55.8 \%$ of patients showed a falling trend in ferritin levels. Patients on subcutaneous desferrioxamine had higher ferritin levels compared to those only on deferasirox (4950 vs. $2990 \mathrm{ng} / \mathrm{dl}, \mathrm{t}=2.3, \mathrm{p}=0.021$ ).

Table 3: Distribution of serum ferritin levels among TDT patients ( $n=52)$

\begin{tabular}{|l|c|}
\hline Serum ferritin range (ng/d) & $\mathbf{n}(\mathbf{\%})$ \\
\hline 1000 or less & $02(03.8)$ \\
\hline $1001-2500$ & $26(50.0)$ \\
\hline $2501-5000$ & $13(25.0)$ \\
\hline More than 5000 & $11(21.2)$ \\
\hline
\end{tabular}

Figure 1 illustrates the correlation between serum ferritin values and non-adherence rates; there is a statistically significant positive correlation $(\mathrm{r}=0.339,95 \% \mathrm{CI}$ : 0.071-0.606, $\mathrm{p}=0.014)$.

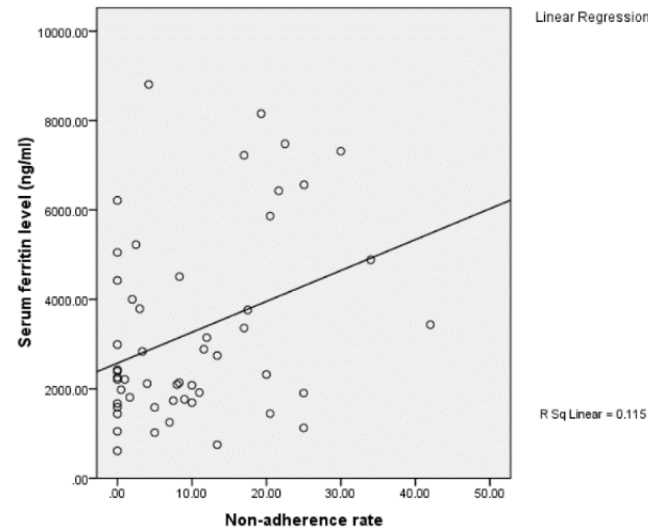

Figure 1: Relationship between serum ferritin level and non-adherence rate (regression line included)

Psychological profile of patients and mothers
On a scale of 0 to 40, patients' SDQ scores ranged from 0 to 22; the mean SDQ score of the 52 patients was 9.9 points $(\mathrm{SD}=4.8)$. Based on cut-off points for normal, borderline and clinical ranges of emotional and behavioural problems, 3 patients $(5.8 \%)$ and 6 patients $(11.5 \%)$ fell into clinical and borderline ranges respectively. GHQ-30 was completed and returned by 49 parents. Parental GHQ scores ranged from 0 to 58 , the mean being 26.5 points $(\mathrm{SD}=15)$.

\section{Psychosocial correlates of non-adherence and serum ferritin levels}

Results from univariate and multivariate analyses of predictors of non-adherence percentage are summarized in Table 4. A significant univariate association was observed between older patient age and poor adherence. Significance of this association disappeared when adjusted for other variables; none of the 7 predictor variables fitted in this model showed statistically significant independent contributions to non-adherence. This model was a poor fit, with an adjusted $\mathrm{R}$ square of $0.064(\mathrm{p}=0.2)$.

Table 4: Adjusted and unadjusted relationships between predictor variables and adherence rates $(n=49)$

\begin{tabular}{|l|c|c|c|c|}
\hline \multirow{2}{*}{ Variable } & \multicolumn{2}{c|}{ Unadjusted univariate analysis } & \multicolumn{2}{c|}{ Adjusted linear regression analysis } \\
\cline { 2 - 5 } & Beta (SE) & p value & Beta (SE) & p value \\
\hline Age & $-0.4(0.2)$ & $0.046^{*}$ & $0.25(0.23)$ & 0.288 \\
\hline Sex & $4.2(3)$ & 0.166 & $-5(3.2)$ & 0.126 \\
\hline Mother's education & $-0.62(0.3)$ & 0.082 & $-0.8(0.6)$ & 0.168 \\
\hline Father's education & $0.15(0.5)$ & 0.752 & $0.8(0.7)$ & 0.281 \\
\hline Family income & $-0.007(0.01)$ & 0.356 & $0.0003(0.001)$ & 0.693 \\
\hline SDQ total score & $0.3(0.3)$ & 0.314 & $-0.35(0.32)$ & 0.277 \\
\hline GHQ-30 score & $-0.08(0.1)$ & 0.414 & $0.16(0.1)$ & 0.131 \\
\hline
\end{tabular}

Note: Unstandardized beta coefficients (Standard errors) and $p$ values from both univariate and multivariate linear regressions are presented. For sex, female $=0$, male $=1$. Adjusted $R$ square for the multiple linear regression is $0.064(p=0.206)$ indicating a poor model fit. ${ }^{*} p<0.05$

Multivariate analysis of psychosocial predictors of serum ferritin levels revealed that increasing age and lower SDQ score of the patient, and lower educational level and greater GHQ-30 score of the mother were independent predictors of greater iron burden. Gender, father's educational level and parental income were not associated. This model showed a good fit, because $42 \%$ of the variance of serum ferritin levels could be explained by the above variables. Table 5 summarizes these regression results along with unadjusted univariate relationships. 
Table 5: Adjusted and unadjusted relationships between predictor variables and serum ferritin levels $(N=49)$

\begin{tabular}{|l|c|c|c|c|}
\hline \multirow{2}{*}{ Variable } & \multicolumn{2}{c|}{ Unadjusted univariate analysis } & \multicolumn{2}{c|}{ Adjusted multivariate analysis } \\
\cline { 2 - 5 } & Beta (SE) & p value & Beta (SE) & p value \\
\hline Age & $114(36)$ & $0.003^{*}$ & $77(37)$ & $0.043^{*}$ \\
\hline Sex & $297(620)$ & 0.633 & $-100(508)$ & 0.845 \\
\hline Mother's education & $-283(63)$ & $<0.001^{*}$ & $-360(91)$ & $<0.001^{*}$ \\
\hline Father's education & $-214(97)$ & $0.033^{*}$ & $170(116)$ & 0.153 \\
\hline Family income & $-0.011(0.016)$ & 0.486 & $0.022(0.016)$ & 0.167 \\
\hline SDQ total score & $-118(59)$ & 0.053 & $-105(58)$ & $0.048^{*}$ \\
\hline GHQ-30 score & $16.8(20)$ & 0.426 & $38(18)$ & $0.030^{*}$ \\
\hline
\end{tabular}

Note: Unstandardized beta coefficients (Standard errors) and p values from both univariate and multivariate linear regressions are presented. For sex, female=0, male=1. Adjusted $R$ square for the multiple linear regression is $0.422(p<0.001)$ indicating a good model fit. ${ }^{*} p<0.05$

\section{Discussion}

Despite advances in its assessment and treatment, iron overload poses an unrelenting challenge to the clinicians managing TDT patients ${ }^{1}$. Bone marrow transplantation, the only curative treatment avenue for TDT, remains unavailable and unaffordable for most patients in Sri Lanka ${ }^{4}$. Therefore, TDT patients in Sri Lanka rely on medical management involving blood transfusions and iron chelation therapy. In our centre, serum ferritin levels are monitored three monthly and dose escalation done accordingly. Despite such monitoring protocols, a dismayingly high degree of iron burden was revealed in this study. Whilst it is generally recommended to maintain serum ferritin levels below $2500 \mathrm{ng} / \mathrm{dl}$, almost half $(46.2 \%)$ of patients in this centre showed levels exceeding this range ${ }^{1}$. Some evidence recommends maintaining ferritin levels even below $1000 \mathrm{ng} / \mathrm{dl}$ to achieve further clinical advantages ${ }^{17}$. Only $3.8 \%$ of patients in our centre were in this range.

There are several limitations to the utility of ferritin in monitoring of iron burden, to be borne in mind. It may rise in the context of inflammation or liver damage, falsely indicating inadequacy of chelation therapy ${ }^{18}$. Moreover, a non-linear relationship between serum ferritin level and body iron stores has been observed at higher ferritin levels ${ }^{19}$.

Adherence rates reported in this study are inadequate, given the prognostic implications. The actual adherence rates may be even lower as participants might have overstated adherence levels. Higher adherence rates have been reported previously for deferasirox in certain developed countries; mean adherence rates from Spain and USA were $92 \%$ and $97 \%$ respectively ${ }^{20,21}$. In the latter study, 90\% adherence was reported by $90 \%$ of patients while in the former study, $55 \%$ of patients had maintained $90 \%$ adherence throughout the duration of follow up. In comparison, $61.6 \%$ of patients in the present study claimed to be at least $90 \%$ adherent. Lower rates have also been reported elsewhere; the overall rate of adherence in Jordan was $73 \%{ }^{22}$. However, what levels of adherence amount to "good adherence" and "poor adherence" remain unclear" ${ }^{11}$.

Only a handful of studies have explored psychosocial factors underlying medication adherence in $\mathrm{TDT}^{11}$. The available few studies have consistently demonstrated an association between older age and poor adherence ${ }^{11}$. As medication adherence among younger children is more a function of parental involvement than children's compliance, adherence rates are likely to be better among younger patients.

Mother's level of education being the most significant predictor of good adherence reflects the pivotal role played by mothers of TDT patients in their care. Despite recent changes in sex roles of parents in child care mainly in Western cultures, mothers still shoulder much of the practical burden of caring for children in Asian cultures like Sri Lanka, while fathers focus on earning a living ${ }^{23}$.

Mental health measures of both the parent and the patient were significantly associated with chelation adequacy. The association between poor mental health of the parent and non-adherence may be bidirectional; parents with mental health issues may not be able to properly attend to their children and conversely, caring for patients with poor adherence and iron overload complications would be taxing and lead to greater parental distress.

Greater levels of mental health problems among children being associated with better compliance seems counterintuitive. However, a similar observation has been made previously in Turkey as well ${ }^{24}$. We postulate two theories to explain this phenomenon. On the one hand, parents of children with more behavioural issues may get more involved with the medical care of their children, leading to better chelation adequacy. On the other hand, children who are more conscious about their disease may adhere to treatment better, despite having greater psychological distress. Since 17.3\% of TDT children demonstrated considerable mental health problems, it is important that they receive 
appropriate psychological support. Screening these patients for psychological issues at regular intervals, and referring to mental health services accordingly, would improve their quality of life. It should also be noted that although the SDQ, the measure selected to assess patients' mental health, has been tested on young adults previously, the interpretation of its scores in this older age range is contentious $^{14}$. However, it is generally observed that TDT patients who are followed up at paediatric wards are a relatively homogenous group in this respect.

Although a positive correlation was demonstrated between serum ferritin and non-adherence levels, the strength of this correlation is not very marked. An important consideration in this respect is the questionable efficacy of the drug formulations available in Sri Lanka. Deferasirox, the only oral chelation drug available in the state sector, comes in a dispersible tablet form which requires dissolution in an appropriate liquid prior to ingestion. It should be taken early in the morning at least 30 minutes prior to a meal. Even though, over the recent years, film-coated tablet formulations with better tolerability and bioavailability have been introduced, they are not available in the state sector in Sri Lanka ${ }^{25}$. Making more tolerable formulations of deferasirox available may therefore be another important future step towards improving medication adherence among TDT patients.

The small sample size of this study is a major limitation to drawing inferences about TDT patients. Adherence rates reported by patients and mothers are likely to be biased, and should be interpreted cautiously. Some of the relationships investigated in the study, such as the relationship between non-adherence and ferritin levels, and the factors associated with non-adherence, may be affected by many confounding variables not studied herein. Therefore, the conclusion that psychosocial factors significantly predict nonadherence is not robust.

Unacceptably high serum ferritin levels and poor medication adherence envisage poor long-term outcomes for TDT patients. Older age and better mental health status of the patient, as well as lower educational level and poor mental health status of the mother were independent predictors of inadequate iron chelation. Interventions to improve drug adherence should therefore target older children and mothers with lower educational level. Evaluating and addressing the mental health issues of parents and TDT patients are also recommended. As patients seem to poorly tolerate the available chelation tablets, making more tolerable formulations available may potentially improve adherence. Future research, enrolling patients from multiple centres and expanding upon the psychosocial and drug regimen-related predictors, is recommended.

\section{Conclusions}

In the Paediatric Unit of National Hospital, Kandy, $46 \%$ patients had serum ferritin (SF) levels $>2500$ $\mathrm{ng} / \mathrm{ml}$. There was a significant correlation between $\mathrm{SF}$ levels and medication non-adherence $(\mathrm{r}=0.34$, $\mathrm{p}=0.014)$. On multivariate analysis, increasing age and lower SDQ score of the patient, lower educational level and greater GHQ-30 score of the mother were independent predictors of higher SF levels, but not of adherence rates.

\section{Acknowledgements}

We thank Sachini Arunarathne, Sanura Vithana, Eranga Jayasinghe and Gayan Amarasinghe for their contributions to data collection.

\section{References}

1. Cappellini MD, Cohen A, Porter J, Taher A, Viprakasit V. Guidelines for the management of transfusion dependent thalassaemia. 3rd edition. Thalassaemia International Federation; 2014.

2. Modell B. Bulletin of the World Health Organization:Global epidemiology of haemoglobin disorders and derived service indicators [Internet]. World Health Organization. 2008 [cited 2019 Sep 20]. Available from: https://www.who.int/bulletin/volumes/86/ 6/06-036673/en/

https://doi.org/10.2471/BLT.06.036673

PMid: 18568278 PMCid: PMC2647473

3. Premawardhana AP, Mudiyanse R, De Silva ST, Jiffry N, Nelumdeniya U, de Silva U, et al. A nationwide survey of hospital-based thalassaemia patients and standards of care and a preliminary assessment of the national prevention program in Sri Lanka. PLoS One 2019; 14(8): $\mathrm{e} 0220852$.

https://doi.org/10.1371/journal.pone.0220 852

PMid: 31419232 PMCid: PMC6697367

4. de Silva S, Fisher CA, Premawardhena A, Lamabadusuriya SP, Peto TE, Perera G, et al. Thalassaemia in Sri Lanka: implications for the future health burden of Asian populations. Sri Lanka Thalassaemia Study Group. Lancet 2000; 355(9206): 786-91. https://doi.org/10.1016/S01406736(99)08 246-X 
5. Ladis V, Chouliaras G, Berdousi H, Kanavakis E, Kattamis C. Longitudinal study of survival and causes of death in patients with thalassemia major in Greece. Annals of the New York Academy of Sciences 2005; 1054:445-50. https://doi.org/10.1016/S01406736(99)08 246-X

6. Kontoghiorghes GJ, Aldouri MA, Sheppard L, Hoffbrand AV. 1,2-dimethyl3-hydroxypyrid-4-one, an orally active chelator for treatment of iron overload. Lancet 1987; 1(8545):1294-5. https://doi.org/10.1016/S01406736(87)90 545-9

7. Cappellini MD, Cohen A, Piga A, Bejaoui M, Perrotta S, Agaoglu L, et al. A phase 3 study of deferasirox (ICL670), a oncedaily oral iron chelator, in patients with beta-thalassemia. Blood 2006; 107(9): 3455-62.

https://doi.org/10.1182/blood-2005-08-

3430

PMid: 16352812

8. Ajebo G, Mangaonkar AA, Ahmad I, Barrett N, Wells L, Patel N, et al. Correlation of serum ferritin levels with liver iron concentration by MRI measurement in sickle cell patients with transfusional iron overload. Blood 2018; 132(Suppl 1): 4926.

https://doi.org/10.1182/blood-2018-99119597

9. Olivieri NF, Nathan DG, MacMillan JH, Wayne AS, Liu PP, McGee A, et al. Survival in medically treated patients with homozygous beta-thalassemia. New England Journal of Medicine 1994; 331(9):574-8.

https://doi.org/10.1056/NEJM1994090133 10903

PMid: 8047081

10. Panda K, Mishra NR, Jena SK. Adherence to deferasirox among beta-thalassemia major children - A cross-sectional study in a tertiary care hospital. Indian Journal of Child Health 2018; 5(1):

11. Evangeli M, Mughal K, Porter JB. Which psychosocial factors are related to chelation adherence in thalassemia? A systematic review. Hemoglobin 2010; 34(3):305-21.

https://doi.org/10.3109/03630269.2010.48
5080

PMid: 20524820

12. Patel P, Beamish $P$, da Silva T, Kaushalya $\mathrm{D}$, Premawardhena A, Williams $\mathrm{S}$, et al. Examining depression and quality of life in patients with thalassaemia in Sri Lanka. International Journal of Noncommunicable Diseases 2019; 4(1): 27-33.

https://doi.org/10.4103/jncd.jncd_49_18

13. Perera S, Thalagala E, Chandrarathna SH, Agampodi TC, Nugegoda DB, Agampodi $\mathrm{SB}$. Factor structure and normative data of the Sinhalese version of self reported Strength and Difficulties Questionnaire (SDQ) for adolescents. Ceylon Medical Journal 2013; 58(2):66-71. https://doi.org/10.4038/cmj.v58i2.5682 PMid: 23817936

14. Brann P, Lethbridge MJ, Mildred H. The young adult Strengths and Difficulties Questionnaire (SDQ) in routine clinical practice. Psychiatry Research 2018; 264:340-5.

https://doi.org/10.1016/j.psychres.2018.03 .001

PMid: 29674224

15. Byerly MJ, Nakonezny PA, Rush AJ. The Brief Adherence Rating Scale (BARS) validated against electronic monitoring in assessing the antipsychotic medication adherence of outpatients with schizophrenia and schizoaffective disorder. Schizophrenia Research 2008; 100(1-3):60-9.

https://doi.org/10.1016/j.schres.2007.12.4 70

PMid: 18255269

16. Abeysena $\mathrm{C}$, Jayawardana $\mathrm{P}$, Peiris U. Factor structure and reliability of the 30item Sinhala version of General Health Questionnaire (GHQ). Asian Journal of Psychiatry 2012; 5(2):150-3. https://doi.org/10.1016/j.ajp.2012.02.025 PMid: 22813658

17. Borgna-Pignatti C, Rugolotto S, De Stefano P, Zhao H, Cappellini MD, Del Vecchio GC, et al. Survival and complications in patients with thalassemia major treated with transfusion and deferoxamine. Haematologica 2004; 89(10):1187-93. 
18. Adamkiewicz TV, Abboud MR, Paley C, Olivieri N, Kirby-Allen M, Vichinsky E, et al. Serum ferritin level changes in children with sickle cell disease on chronic blood transfusion are nonlinear and are associated with iron load and liver injury. Blood [Internet] 2009; 114(21):4632.

https://doi.org/10.1182/blood-2009-02203323

PMid: 19721013 PMCid: PMC2780299

19. Porter JB, Elalfy M, Taher A, Aydinok Y, Lee S-H, Sutcharitchan $\mathrm{P}$, et al. Limitations of serum ferritin to predict liver iron concentration responses to deferasirox therapy in patients with transfusion-dependent thalassaemia. European Journal of Haematology 2017; 98(3):280-8.

https://doi.org/10.1111/ejh.12830

PMid: 27859648

20. Trachtenberg F, Vichinsky E, Haines D, Pakbaz Z, Mednick L, Sobota A, et al. Iron chelation adherence to deferoxamine and deferasirox in thalassaemia. American Journal of Hematology 2011; 86(5):4336. https://doi.org/10.1002/ajh.21993 PMid: 21523808 PMCid: PMC4599708

21. Escudero-Vilaplana V, Garcia-Gonzalez $\mathrm{X}$, Osorio-Prendes S, Romero-Jimenez RM, Sanjurjo-Saez M. Impact of medication adherence on the effectiveness of deferasirox for the treatment of transfusional iron overload in myelodysplastic syndrome. Journal of Clinical Pharmacy and Therapeutics 2016; 41(1): 59-63. https://doi.org/10.1111/jcpt.12348

PMid: 26778738

22. Al-Kloub MI, A Bed MA, Al Khawaldeh OA, Al Tawarah YM, Froelicher ES. Predictors of non-adherence to follow-up visits and deferasirox chelation therapy among Jordanian adolescents with thalassaemia major. Pediatric Hematology and Oncology 2014; 31(7):624-37.

https://doi.org/10.3109/08880018.2014.93 9792

PMid: 25116329

23. Banchefsky S, Park B. The "New Father": Dynamic Stereotypes of Fathers. Psychology of Men and Masculinity 2015; 17(1). https://doi.org/10.1037/a0038945

24. Aydinok Y, Erermis S, Bukusoglu N, Yilmaz D, Solak U. Psychosocial implications of thalassaemia major. Pediatric International 2005; 47(1):84-9. https://doi.org/10.1111/j.1442200x.2004.0 2009.x

PMid: 15693873

25. Taher AT, Origa R, Perrotta S, Kourakli A, Ruffo GB, Kattamis A, et al. New filmcoated tablet formulation of deferasirox is well tolerated in patients with thalassemia or lower-risk MDS: Results of the randomized, phase II ECLIPSE study. American Journal of Hematology 2017; 92(5):420-8.

https://doi.org/10.1002/ajh.24668

PMid: 28142202 PMCid: PMC6585741 